\title{
Fixed Gain Amplify-and-Forward Relaying with Co-Channel Interference
}

\author{
Himal A. Suraweera*, Diomidis S. Michalopoulos ${ }^{\dagger}$, Robert Schober ${ }^{\dagger}$, George K. Karagiannidis ${ }^{\ddagger}$, and Arumugam Nallanathan ${ }^{\S}$ \\ *Engineering Product Development, Singapore University of Technology and Design, Singapore (e-mail: himalsuraweera@sutd.edu.sg) \\ $\dagger$ Department of Electrical \& Computer Engineering, The University of British Columbia, Canada (e-mail:\{dio, rschober\}@ece.ubc.ca)

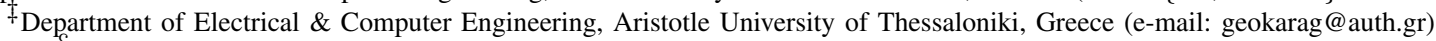

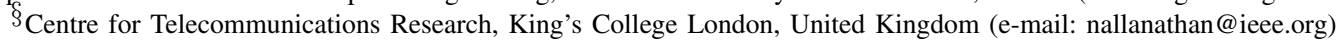

\begin{abstract}
We investigate the outage probability and the average bit error rate (BER) of a dual-hop fixed gain relaying system in the presence of co-channel interference and thermal noise at the relay and the destination. Our analysis assumes Rayleigh fading for the source-relay and relay-destination channels and Rician fading for the interfering channels. We present new closed-form/series expressions for the outage probability and the average BER, which allow for a rapid performance evaluation of fixed-gain relaying in interference-impaired environments. The achievable diversity order is also studied. It is further shown through numerical results that the Rician $K$-factor of the interfering channel has a negative effect on the overall outage probability and BER, yet this effect is very small.
\end{abstract}

\section{INTRODUCTION}

Amplify-and-forward (AF) relaying has now become a well studied protocol under thermal noise-limited conditions, (see e.g. [1]-[3] and references therein). However, in practice, the interference present in relay networks can cause severe performance degradation [4].

Several recent works have studied the performance of $\mathrm{AF}$ and decode-and-forward (DF) relaying for interference-limited (negligible thermal noise) relay(s) or destination(s), see e.g. [4]-[10]. In [4], the impact of interference on the performance of a dual-hop channel state information (CSI)-assisted AF relay network has been investigated. The authors of [5] have analyzed the performance gains of a half-duplex multi-user network where the relay-destination slot is reused, causing interference at the destination nodes. In [6], [7], the performance of different DF relaying systems in the presence of interference has been investigated. Assuming an interference-limited destination, in [8], the outage probability of a fixed gain AF relay system over Rayleigh fading channels has been investigated. In [9], the performance of a dual-hop CSI-assisted AF system with interference-limited relays has been studied. In [10], the outage probability of dual-hop CSI-assisted AF relaying with interference-limited relays and destinations has been derived.

In cellular applications, the case of interference at the relay has practical significance since relays may operate at the cell edge providing extended coverage. Thus, due to the reuse of frequency bands it is likely that relays are affected by lineof-sight $(\mathrm{LoS}) /$ non LoS co-channel interference and thermal noise. Nonetheless, the joint effects of interference and thermalnoise on the performance of dual-hop fixed gain relay networks has not been investigated in the existing literature. Motivated by the absence of such an analysis, in this paper, we study the outage probability and the average bit error rate (BER) of dual-hop fixed gain AF relaying with interference and thermal noise at the relay. In addition, the case where there exists interference at the destination is also considered, and analyzed separately. Assuming Rayleigh fading for the source-relay and relay-destination channels and Rician fading for the interfering channel, we derive new outage and BER expressions upon statistically characterizing the signal-to-interference plus noise ratio (SINR). The Rician fading assumption on the interference link is motivated by the fact that it is used to model wireless propagation comprising a LoS component and a scattered component. Therefore, the derived analytical expressions quantify the impact of interference on relay system performance for a large set of environments. The analysis of Rician channels is generally more difficult and as a special case, includes the commonly assumed Rayleigh fading.

\section{SySTEM MODEL}

\section{A. Mode of Operation}

We consider a communication system, where a source, $S$, communicates with a destination, $D$, through a relay, $R$. All nodes are equipped with a single antenna. It is assumed that $S$ does not have a direct link to $D$. The communication in the system is divided into two orthogonal time intervals. In the first time interval, $S$ sends its symbol $s_{0}$ to $R$. In the second time interval, $R$ communicates with $D$.

At $R$, the received signal in the presence of a single interferer, with an average power, $P_{1}$, and additive white Gaussian noise (AWGN) can be expressed as

$$
y_{r}=\sqrt{P_{s}} h_{s r} e^{j \theta_{s r}} s_{0}+\sqrt{P_{1}} h_{1} e^{j \theta_{1}} s_{1}+n_{r},
$$

where $P_{s}$ is the transmit power, $s_{1}$ is the interference symbol and $h_{1}$ is the channel amplitude of an interferer located in the proximity of $R ; h_{s r}$ and $h_{r d}$ are the Rayleigh fading amplitudes of the $S-R$ and $R-D$ links, respectively, with average powers $E\left[\left|h_{s r}\right|^{2}\right]=\sigma_{s r}^{2}$ and $E\left[\left|h_{r d}\right|^{2}\right]=\sigma_{r d}^{2}$, respectively, with $E[\cdot]$ denoting expectation. The phase of the useful and the interfering signal at $R$, are denoted by $\theta_{s r}$ and $\theta_{1}$, respectively. It is further assumed that $h_{1}$ is a Rician distributed random variable (RV) with $E\left[\left|h_{1}\right|^{2}\right]=\sigma_{1}^{2}$, and is independent of $h_{s r}$ and $h_{r d}$. Finally, $n_{r}$ denotes the AWGN at $R$ satisfying $E\left[\left|n_{r}\right|^{2}\right]=N_{01}$.

\section{B. Considered Scenarios}

Depending on whether or not there exists interference at $D$, two cases are considered in this paper which are referred to as 
Scenario (a) and Scenario (b), respectively. Specifically:

- Scenario (a): $R$ experiences interference and AWGN while $D$ is interference-free and subject to AWGN only. Reference [4] first considered Scenario (a) in an analysis of CSI-assisted AF relays.

- Scenario (b): $R$ experiences interference and AWGN while $D$ is interference-limited. An interference-limited $D$ was also considered in [8], however, no interference was assumed at $R$.

1) Scenario (a): Let $\mathcal{G}_{\mathrm{F}}$ denote the fixed gain employed by $R$. In the absence of interference at $D$, the received signal can be expressed as

$y_{d}=h_{r d} e^{j \theta_{r d}} \mathcal{G}_{\mathrm{F}}\left(\sqrt{P_{s}} h_{s r} e^{j \theta_{s r}} s_{0}+\sqrt{P_{1}} h_{1} e^{j \theta_{1}} s_{1}+n_{r}\right)+n_{d}$

where $n_{d}$ is the AWGN at $D$ satisfying $E\left[\left|n_{d}\right|^{2}\right]=N_{02}$, and $\theta_{r d}$ denotes the phase of the $R-D$ channel. Therefore, we obtain the overall SINR as

$$
\gamma_{e q 1}=\frac{P_{s}\left|h_{s r}\right|^{2}\left|h_{r d}\right|^{2}}{\left|h_{r d}\right|^{2} N_{01}+P_{1}\left|h_{r d}\right|^{2}\left|h_{1}\right|^{2}+\frac{N_{02}}{\mathcal{G}_{\mathrm{F}}^{2}}} .
$$

2) Scenario (b): The received signal at $D$ is given by

$$
\begin{aligned}
y_{d} & =h_{r d} e^{j \theta_{r d}} \mathcal{G}_{\mathrm{F}}\left(\sqrt{P_{s}} h_{s r} e^{j \theta_{s r}} s_{0}+\sqrt{P_{1}} h_{1} e^{j \theta_{1}} s_{1}\right) \\
& +h_{r d} e^{j \theta_{r d}} \mathcal{G}_{\mathrm{F}} n_{r}+\sqrt{P_{2}} h_{2} e^{j \theta_{2}} s_{2},
\end{aligned}
$$

where $h_{2}$ is the Rician faded channel from an interferer located in the proximity of $D$ with average power $E\left[\left|h_{2}\right|^{2}\right]=\sigma_{2}^{2} ; s_{2}$ is the signal from the interferer in the proximity of $D$ and $\theta_{2}$ denotes the phase of the interfering channel at $D$. The overall SINR can be thus expressed as

$$
\gamma_{e q 2}=\frac{P_{s}\left|h_{s r}\right|^{2}\left|h_{r d}\right|^{2}}{\left|h_{r d}\right|^{2} N_{01}+P_{1}\left|h_{r d}\right|^{2}\left|h_{1}\right|^{2}+\frac{P_{2}\left|h_{2}\right|^{2}}{\mathcal{G}_{\mathrm{F}}^{2}}}
$$

\section{Outage Probability Analysis}

In this section, the outage probability under Scenarios $(a)$ and (b) is studied. In the case of outage probability, it is reasonable to assume that $s_{0}, s_{1}$ and $s_{2}$ are complex Gaussian distributed RVs with unity average power. Since the relay's output power is constrained, $\mathcal{G}_{\mathrm{F}}$ takes the form:

$$
\mathcal{G}_{\mathrm{F}}=\sqrt{\frac{P_{r}}{P_{s} \sigma_{s r}^{2}+P_{1} \sigma_{1}^{2}+N_{01}}} .
$$

1) Scenario (a): Using (6) we can simplify (3) as

$$
\gamma_{e q 1}=\frac{\gamma_{1} \gamma_{2}}{(1+u) \gamma_{2}+C}
$$

where $\gamma_{1}=\frac{P_{s}}{N_{01}}\left|h_{s r}\right|^{2}, \gamma_{2}=\frac{P_{r}}{N_{02}}\left|h_{r d}\right|^{2}$, and $u=\frac{P_{1}}{N_{01}}\left|h_{1}\right|^{2}$. The constant $C=\bar{\gamma}_{1}+\eta_{1}+1$, where we have defined $\bar{\gamma}_{1}=\frac{P_{s} \sigma_{s r}^{2}}{N_{01}}$ and $\eta_{1}=\frac{P_{1} \sigma_{1}^{2}}{N_{01}}$.

To derive the outage probability of $\gamma_{e q 1}$, conditioning on $\gamma_{2}$ and $u$, we first express the cumulative distribution function (cdf)

$$
\begin{aligned}
& \text { of } \gamma_{e q 1} \text { as } \\
& F_{\gamma_{e q 1}}\left(\gamma_{T}\right)=\operatorname{Pr}\left\{\frac{\gamma_{1} \gamma_{2}}{(1+u) \gamma_{2}+C}<\gamma_{T}\right\} \\
& =\int_{0}^{\infty} \int_{0}^{\infty} \operatorname{Pr}\left\{\gamma_{1}<(1+x) \gamma_{T}+\frac{C \gamma_{T}}{y}\right\} f_{u}(x) f_{\gamma_{2}}(y) d x d y
\end{aligned}
$$

where $\operatorname{Pr}\{\cdot\}$ denotes probability and $\gamma_{T}$ represents the outage threshold SNR. The cdf of $\gamma_{1}, F_{\gamma_{1}}(x)$ and the probability density function (pdf) of $\gamma_{2}, f_{\gamma_{2}}(x)$ are given by $F_{\gamma_{1}}(x)=1-$ $e^{-\frac{x}{\bar{\gamma}_{1}}}$ and $f_{\gamma_{2}}(x)=\frac{1}{\bar{\gamma}_{2}} e^{-\frac{x}{\bar{\gamma}_{2}}}$, respectively, where $\bar{\gamma}_{2}=\frac{P_{r} \sigma_{r d}^{2}}{N_{02}}$. Since the interferer at $R$, is subject to Rician fading, the pdf of $u$ is given by

$$
f_{u}(x)=\frac{\left(1+\omega_{1}\right) e^{-\omega_{1}-\frac{\left(1+\omega_{1}\right) x}{\eta_{1}}}}{\eta_{1}} I_{0}\left(2 \sqrt{\frac{\omega_{1}\left(1+\omega_{1}\right) x}{\eta_{1}}}\right)
$$

where $\omega_{1}$ is the Rician $K$-factor, defined as the ratio of the powers of the LoS component to the scattered components, and $I_{0}(\cdot)$ is the zeroth order modified Bessel function of the first kind defined in [11, Eq. (8.431.1)].

Substituting the pdfs and the cdf into (8) we obtain

$$
\begin{aligned}
& F_{\gamma_{e q 1}}\left(\gamma_{T}\right)=1-\frac{\left(1+\omega_{1}\right) e^{-\omega_{1}-\frac{\gamma_{T}}{\bar{\gamma}_{1}}}}{\eta_{1} \bar{\gamma}_{2}} \int_{0}^{\infty} e^{-\left(\frac{1+\omega_{1}}{\eta_{1}}+\frac{\gamma_{T}}{\bar{\gamma}_{1}}\right) x} \\
& \times I_{0}\left(2 \sqrt{\frac{\omega_{1}\left(1+\omega_{1}\right) x}{\eta_{1}}}\right) d x \int_{0}^{\infty} e^{-\frac{C \gamma_{T}}{\bar{\gamma}_{1} y}-\frac{y}{\gamma_{2}}} d y
\end{aligned}
$$

Using [11, Eq. (3.471.9)] and [12, Eq. (9)]

$$
\int_{0}^{\infty} x e^{-\frac{p^{2} x^{2}}{2}} I_{0}(a x) d x=\frac{1}{p^{2}} e^{\frac{a^{2}}{2 p^{2}}}
$$

in (10) yields

$$
\begin{aligned}
F_{\gamma_{e q 1}}\left(\gamma_{T}\right) & =1-2\left(1+\omega_{1}\right) \frac{e^{-\omega_{1}-\frac{\gamma_{T}}{\bar{\gamma}_{1}}+\frac{\omega_{1}}{1+\frac{\eta 1 \gamma_{T}}{\left(1+\omega_{1}\right) \bar{\gamma}_{1}}}}}{1+\omega_{1}+\frac{\eta_{1} \gamma_{T}}{\bar{\gamma}_{1}}} \\
& \times \sqrt{\frac{C \gamma_{T}}{\bar{\gamma}_{1} \bar{\gamma}_{2}}} K_{1}\left(2 \sqrt{\frac{C \gamma_{T}}{\bar{\gamma}_{1} \bar{\gamma}_{2}}}\right)
\end{aligned}
$$

where $K_{1}(\cdot)$ is the first order modified Bessel function of the second kind defined in [11, Eq. (8.432.6)]. As expected, we notice in (12) that the existence of interference at the relay increases the overall outage probability. Furthermore, taking the first order derivative of (12) with respect to $\omega_{1}$ we obtain

$$
\begin{aligned}
\frac{\partial F_{\gamma_{e q 1}}\left(\gamma_{T}\right)}{\partial \omega_{1}} & =2\left(\eta_{1} \gamma_{T}\right)^{2} \sqrt{\frac{C \bar{\gamma}_{1} \gamma_{T}}{\bar{\gamma}_{2}}} K_{1}\left(2 \sqrt{\frac{C \gamma_{T}}{\bar{\gamma}_{1} \bar{\gamma}_{2}}}\right) \\
& \times \frac{\omega_{1}}{\left(\left(1+\omega_{1}\right) \bar{\gamma}_{1}+\eta_{1} \gamma_{T}\right)^{3}} e^{-\omega_{1}-\frac{\gamma_{T}}{\bar{\gamma}_{1}}+\frac{\omega_{1}}{1+\frac{\omega_{1} \gamma_{T}}{\left(1+\omega_{1}\right) \bar{\gamma}_{1}}}} .
\end{aligned}
$$

Since $\frac{\partial F_{\gamma_{e q 1}}\left(\gamma_{T}\right)}{\partial \omega_{1}}>0$ holds, we infer that the outage probability is an increasing function of $\omega_{1}$. As a result, an interferer with a high Rician $K$-factor increases the outage probability, as compared to an interferer with a low Rician $K$-factor.

2) Scenario (b): In this scenario, we can express the overall SINR as

$$
\gamma_{e q 2}=\frac{\gamma_{1} \gamma_{2}}{(1+u) \gamma_{2}+C v}
$$


where $v=\frac{P_{2}}{N_{02}}\left|h_{2}\right|^{2}$.

The outage probability can be expressed as

$$
\begin{aligned}
& F_{\gamma_{e q 2}}\left(\gamma_{T}\right)=\operatorname{Pr}\left(\frac{\gamma_{1} \gamma_{2}}{(1+u) \gamma_{2}+C v}<\gamma_{T}\right) \\
& =\int_{0}^{\infty} \int_{0}^{\infty} \int_{0}^{\infty} \operatorname{Pr}\left(\gamma_{1}<(1+x) \gamma_{T}+\frac{C \gamma_{T} z}{y}\right) \\
& \times f_{u}(x) f_{\gamma_{2}}(y) f_{v}(z) d x d y d z .
\end{aligned}
$$

Since the interferer at $D$ is subject to Rician fading, the pdf of $v$ can be expressed as

$$
f_{v}(x)=\frac{\left(1+\omega_{2}\right) e^{-\omega_{2}-\frac{\left(1+\omega_{2}\right) x}{\eta_{2}}}}{\eta_{2}} I_{0}\left(2 \sqrt{\frac{\omega_{2}\left(1+\omega_{2}\right) x}{\eta_{2}}}\right),
$$

where $\eta_{2}=\frac{P_{2} \sigma_{2}^{2}}{N_{02}}$ and $\omega_{2}$ is the Rician $K$-factor.

Substituting the cdf of $\gamma_{1}$ and the pdfs of $\gamma_{2}, u$, and $v$ we obtain

$$
\begin{aligned}
& F_{\gamma_{e q 2}}\left(\gamma_{T}\right)=1-\frac{\left(1+\omega_{1}\right)\left(1+\omega_{2}\right) e^{-\omega_{1}-\omega_{2}-\frac{\gamma_{T}}{\bar{\gamma}_{1}}}}{\eta_{1} \eta_{2} \bar{\gamma}_{2}} \\
& \times \int_{0}^{\infty} e^{-\left(\frac{1+\omega_{1}}{\eta_{1}}+\frac{\gamma_{T}}{\bar{\gamma}_{1}}\right) x} I_{0}\left(2 \sqrt{\frac{\omega_{1}\left(1+\omega_{1}\right) x}{\eta_{1}}}\right) d x \\
& \times \int_{0}^{\infty} e^{-\frac{\left(1+\omega_{2}\right) z}{\eta_{2}}} I_{0}\left(2 \sqrt{\frac{\omega_{2}\left(1+\omega_{2}\right) z}{\eta_{2}}}\right) \\
& \times \int_{0}^{\infty} e^{-\frac{C \gamma_{T} z}{\bar{\gamma}_{1} y}-\frac{y}{\bar{\gamma}_{2}}} d y d z .
\end{aligned}
$$

With the help of (11) and [11, Eq. (3.471.9)] we solve the integrals with respect to $x$ and $y$ as follows

$$
\begin{aligned}
& F_{\gamma_{e q 2}}\left(\gamma_{T}\right)=1-2 \sqrt{\frac{C \gamma_{T}}{\bar{\gamma}_{1} \bar{\gamma}_{2}}} \frac{\left(1+\omega_{1}\right)\left(1+\omega_{2}\right)}{\left(1+\omega_{1}+\frac{\eta_{1} \gamma_{T}}{\bar{\gamma}_{1}}\right) \eta_{2}} \\
& \times e^{-\omega_{1}-\omega_{2}-\frac{\gamma_{T}}{\bar{\gamma}_{1}}+\frac{\omega_{1}}{1+\frac{\eta_{1} \gamma_{T}}{\left(1+\omega_{1}\right) \bar{\gamma}_{1}}}} \int_{0}^{\infty} \sqrt{z} e^{-\frac{\left(1+\omega_{2}\right) z}{\eta_{2}}} \\
& \times I_{0}\left(2 \sqrt{\frac{\omega_{2}\left(1+\omega_{2}\right) z}{\eta_{2}}}\right) K_{1}\left(2 \sqrt{\frac{C \gamma_{T} z}{\bar{\gamma}_{1} \bar{\gamma}_{2}}}\right) d z .
\end{aligned}
$$

Unfortunately, a closed-form expression for the outage probability of fixed gain relaying under the assumption of Scenario (b) is very difficult, if not impossible, to be obtained. Nonetheless, the integral in (18) can be evaluated in a series form by making the change of variables, $t^{2}=z$, and using [11, Eq. (8.447.1)], [11, Eq. (6.631.3)], [11, Eq. (9.220.4)], [11, Eq. (9.220.2)], and [11, Eq. (9.210.2)], yielding for the case of $\omega_{2} \neq 0$

$$
\begin{aligned}
& F_{\gamma_{e q 2}}\left(\gamma_{T}\right)=1-\frac{\left(1+\omega_{1}\right) e^{-\omega_{1}-\omega_{2}-\frac{\gamma_{T}}{\bar{\gamma}_{1}}+\frac{\omega_{1}}{1+\frac{\eta_{1} \gamma_{T}}{\left(1+\omega_{1}\right) \bar{\gamma}_{1}}}}}{\left(1+\omega_{1}+\frac{\eta_{1} \gamma_{T}}{\bar{\gamma}_{1}}\right)} \\
& \times \sum_{m=0}^{M}(m+1) \omega_{2}^{m} \Psi\left(m+1,0, \frac{C \gamma_{T}}{\left(\frac{1+\omega_{2}}{\eta_{2}}\right) \bar{\gamma}_{1} \bar{\gamma}_{2}}\right)
\end{aligned}
$$

where $\Psi(\cdot, \cdot, \cdot)$ denotes the Tricomi confluent hypergeometric function defined in [11, Eq. (9.210.2)]; $M$ represents the sufficiently large number of terms required for the series in (19) to converge, which in practical cases takes values between one and 20. We also note that for the special case when $h_{2}$ experiences Rayleigh fading $\left(\omega_{2}=0\right)$, using [13, Eq. (2.16.8.5)] in (18) yields

$$
\begin{aligned}
& F_{\gamma_{e q 2}}\left(\gamma_{T}\right)=1-\frac{\left(1+\omega_{1}\right) C \eta_{2} \gamma_{T}}{\left(1+\omega_{1}+\frac{\eta_{1} \gamma_{T}}{\bar{\gamma}_{1}}\right) \bar{\gamma}_{1} \bar{\gamma}_{2}} \\
& \times e^{-\omega_{1}-\left(1-\frac{C \eta_{2}}{\bar{\gamma}_{2}}\right) \frac{\gamma_{T}}{\bar{\gamma}_{1}}+\frac{\omega_{1}}{1+\frac{\eta_{1} \gamma_{T}}{\left(1+\omega_{1}\right) \bar{\gamma}_{1}}} \Gamma\left(-1, \frac{C \eta_{2} \gamma_{T}}{\bar{\gamma}_{1} \bar{\gamma}_{2}}\right)}
\end{aligned}
$$

where $\Gamma(\cdot, \cdot)$ is the complementary incomplete Gamma function defined in [11, Eq. (8.350.2)].

\section{A. Diversity Order}

1) Scenario (a): Using approximation [14, Eq. (9.6.9)] followed by the McLaurin series representation of the exponential function in (12) and taking only the first order terms, (12) yields for high signal-to-noise ratio (SNR)

$$
F_{\gamma_{e q 1}}\left(\gamma_{T}\right) \approx \frac{\gamma_{T}}{\bar{\gamma}_{1}}+\eta_{1} \frac{\gamma_{T}}{\bar{\gamma}_{1}}
$$

Then, expressing $\eta_{1}$ as $\eta_{1}=\mu \bar{\gamma}_{1}$, where $\mu$ is a finite nonzero constant, and using the definition of the diversity order $\left(d=-\lim _{\bar{\gamma}_{1} \rightarrow \infty} \log \left(F_{\gamma_{e q 1}}\left(\gamma_{T}\right)\right) / \log \left(\bar{\gamma}_{1}\right)\right)$ it follows that the diversity order of the interference-limited fixed gain relaying scheme with interference-free reception at $D$, is zero.

2) Scenario (b): For asymptotically high $\bar{\gamma}_{1} / \gamma_{T}$ and for $\omega_{2} \neq 0$, using the approximation $\Psi\left(m+1,0, C \eta_{2} \gamma_{T} /\left[\left(1+\omega_{2}\right) \bar{\gamma}_{1} \bar{\gamma}_{2}\right]\right) \approx 1 /(m+1) \quad$ [14, Eq. (13.5.11)] and the infinite series expansion of the exponential function, (19) yields

$$
F_{\gamma_{e q 2}}\left(\gamma_{T}\right) \approx 1-\frac{\left(1+\omega_{1}\right) e^{-\omega_{1}-\frac{\gamma_{T}}{\bar{\gamma}_{1}}+\frac{\omega_{1}}{1+\frac{\eta_{1} \gamma_{T}}{\left(1+\omega_{1}\right) \bar{\gamma}_{1}}}}}{\left(1+\omega_{1}+\frac{\eta_{1} \gamma_{T}}{\bar{\gamma}_{1}}\right)} .
$$

Taking the first order terms of the Taylor series expansion of (22), we arrive at (21). Therefore, similarly to Section III-A1, it follows that the diversity order of the interference-limited fixed gain relaying scheme with interference-limited reception at $D$, is zero. For the special case of $\omega_{2}=0$, we may infer zero diversity order from (20) and [11, Eq. (8.351.4)].

\section{Average BER AnAlysis}

In this section we derive the system's exact error performance using a characteristic function (CF) method. Moreover, we assume that both the desired signal, $s_{0}$ and the interference signals at $R$ and $D, s_{1}$ and $s_{2}$, are binary phase-shift keying (BPSK) modulated [4]. In the special case of Rayleigh faded interferer/s, we present new exact closed-form BER expressions.

\section{A. Scenario (a)}

After demodulation, matched filtering and sampling, the decision statistic for the data symbol can be expressed as

$\mathcal{D}_{0}=h_{r d} \mathcal{G}_{\mathrm{F}}\left(\sqrt{P_{s}} h_{s r} s_{0}+\sqrt{P_{1}} h_{1} \cos \left(\theta_{1}-\theta_{s r}\right) s_{1}+\tilde{n}_{r}\right)+\tilde{n}_{d}$ 
where $\tilde{n}_{r}$ and $\tilde{n}_{d}$ are zero mean Gaussian RVs with variances $\frac{N_{01}}{2}$ and $\frac{N_{02}}{2}$ respectively. The average BER conditioned on $h_{s r}$ can be written as

$$
\begin{aligned}
\widetilde{P}_{b}=\operatorname{Pr}[ & h_{r d} \mathcal{G}_{\mathrm{F}} \sqrt{P_{s}} h_{s r} s_{0} \\
& \left.+h_{r d} \mathcal{G}_{\mathrm{F}}\left(I+\tilde{n}_{r}\right)+\tilde{n}_{d}<0 \mid s_{0}=+1, h_{s r}\right]
\end{aligned}
$$

where $I=\sqrt{P_{1}} h_{1} \cos \theta s_{1}$ and $\theta=\left(\theta_{1}-\theta_{\text {sr }}\right)$ modulo $2 \pi$. Following [15], [16] we assume that $\theta$ is uniformly distributed over $(0,2 \pi]$. Simplifying further yields

$$
\begin{aligned}
\widetilde{P}_{b}=\operatorname{Pr}[ & \sqrt{P_{s}} h_{s r} s_{0} \\
& \left.+I+\tilde{n}_{r}+\frac{\tilde{n}_{d}}{\mathcal{G}_{\mathrm{F}} h_{r d}}<0 \mid s_{0}=+1, h_{s r}\right] .
\end{aligned}
$$

Let $X_{1}=\tilde{n}_{r}+\frac{\tilde{n}_{d}}{\mathcal{G}_{\mathrm{F}} h_{r d}}$ and $\Lambda=I+X_{1}$. Therefore, (25) can be written as

$$
\widetilde{P}_{b}=\operatorname{Pr}\left(I+W<-\sqrt{P_{s}} h_{s r}\right)=1-F_{\Lambda}\left(\sqrt{P_{s}} h_{s r}\right) .
$$

Since the cdf, $F_{\Lambda}(x)$ can be written as [17, Eq. (3-23)]

$$
F_{\Lambda}(x)=\frac{1}{2}+\frac{1}{\pi} \int_{0}^{\infty} \frac{\sin (x z)}{\omega} \Phi_{\Lambda}(z) d z
$$

where $\Phi_{\Lambda}(z)$ is the $\mathrm{CF}$ of the $\mathrm{RV}, \Lambda$, given by $\Phi_{\Lambda}(z)=$ $\Phi_{I}(z) \Phi_{X_{1}}(z)$. Hence (26) can be re-expressed as

$$
=\frac{1}{2}-\frac{1}{\pi} \int_{0}^{\infty} \frac{\sin \left(\sqrt{P_{s}} r z\right)}{z} \Phi_{\Lambda}(z) d z .
$$

Using the pdf of the Rayleigh distributed RV, $h_{s r}$

$$
f_{h_{s r}}(x)=\frac{2 x}{\sigma_{s r}^{2}} e^{-\frac{x^{2}}{\sigma_{s r}^{2}}},
$$

the unconditional average BER, $P_{b}$ can be written as

$$
P_{b}=\frac{1}{2}-\frac{2}{\pi \sigma_{s r}^{2}} \int_{0}^{\infty} \int_{0}^{\infty} r \sin \left(\sqrt{P_{s}} r z\right) e^{-\frac{r^{2}}{\sigma_{s r}^{2}}} \frac{\Phi_{\Lambda}(z)}{z} d r d z .
$$

Using [11, Eq. (3.952.1)] we can simplify (30) as

$$
P_{b}=\frac{1}{2}\left(1-\sqrt{\frac{P_{s} \sigma_{s r}^{2}}{\pi}} \int_{0}^{\infty} \Phi_{\Lambda}(z) e^{-\frac{P_{s} \sigma_{s s}^{2} z^{2}}{4}} d z\right) .
$$

Now consider $\Phi_{I}(z)$. Using [15, Appendix A] we can write

$$
\Phi_{I}(z)=E\left[e^{j z I}\right]=\int_{0}^{\infty} J_{0}\left(\sqrt{P_{1}} r z\right) f_{h_{1}}(r) d r
$$

where $J_{0}(\cdot)$ is the zeroth order Bessel function of the first kind. Substituting $f_{h_{1}}(r)$ into (32) yields

$$
\begin{aligned}
\Phi_{I}(z) & =\frac{2\left(1+\omega_{1}\right) e^{-\omega_{1}}}{\sigma_{1}^{2}} \int_{0}^{\infty} r e^{-\frac{\left(1+\omega_{1}\right) r^{2}}{\sigma_{1}^{2}}} \\
& \times I_{0}\left(2 r \sqrt{\frac{\omega_{1}\left(1+\omega_{1}\right)}{\sigma_{1}^{2}}}\right) J_{0}\left(\sqrt{P_{1}} r z\right) d r \\
& =e^{-\frac{P_{1} \sigma_{1}^{2} z^{2}}{4\left(1+\omega_{1}\right)}} J_{0}\left(\sqrt{\frac{\omega_{1} P_{1} \sigma_{1}^{2}}{1+\omega_{1}}} z\right)
\end{aligned}
$$

where the last line follows with the help of [11, Eq. (6.633.4)]. Using [11, Eq. (8.432.6)], $\Phi_{X_{1}}(z)$ is expressed as

$$
\begin{aligned}
\Phi_{X_{1}}(z) & =e^{-\frac{N_{01} z^{2}}{4}}\left(\frac{1}{\sigma_{r d}^{2}} \int_{0}^{\infty} e^{-\frac{N_{02} z^{2}}{4 \mathcal{G}_{\mathrm{F}}^{2} h}} e^{-\frac{h}{\sigma_{r d}^{2}}} d h\right) \\
& =\frac{\sqrt{N_{02}}}{\mathcal{G}_{\mathrm{F}} \sigma_{r d}} z e^{-\frac{N_{01} z^{2}}{4}} K_{1}\left(\frac{\sqrt{N_{02}}}{\mathcal{G}_{\mathrm{F}} \sigma_{r d}} z\right) .
\end{aligned}
$$

Hence, substituting (34) and (33) into (31), we obtain

$$
\begin{aligned}
P_{b} & =\frac{1}{2}\left(1-\sqrt{\frac{P_{s} N_{02} \sigma_{s r}^{2}}{\pi \mathcal{G}_{\mathrm{F}}^{2} \sigma_{r d}^{2}}} \int_{0}^{\infty} z e^{-\left(P_{s} \sigma_{s r}^{2}+\frac{P_{1} \sigma_{1}^{2}}{1+\omega_{1}}+N_{01}\right) \frac{z^{2}}{4}}\right. \\
& \left.\times J_{0}\left(\sqrt{\frac{\omega_{1} P_{1} \sigma_{1}^{2}}{1+\omega_{1}}} z\right) K_{1}\left(\frac{\sqrt{N_{02}}}{\mathcal{G}_{\mathrm{F}} \sigma_{r d}} z\right) d z\right) .
\end{aligned}
$$

To the best of the authors' knowledge, Eq. (35) cannot be simplified to a closed-form expression. However, using the following series expansion of $J_{0}(x)$ [11, Eq. (8.402)]

$$
J_{0}(x)=\sum_{m=0}^{\infty} \frac{(-1)^{m}}{(m !)^{2}}\left(\frac{x}{2}\right)^{2 m},
$$

we can write (35) as

$$
\begin{aligned}
P_{b} & =\frac{1}{2}\left(1-\sqrt{\frac{P_{s} N_{02} \sigma_{s r}^{2}}{\pi \mathcal{G}_{\mathrm{F}}^{2} \sigma_{r d}^{2}}} \sum_{m=0}^{\infty} \frac{(-1)^{m}}{(m !)^{2}}\left(\frac{\omega_{1} \eta_{1} N_{01}}{4\left(1+\omega_{1}\right)}\right)^{m}\right. \\
& \left.\times \int_{0}^{\infty} z^{2 m+1} e^{-\left(P_{s} \sigma_{s r}^{2}+\frac{P_{1} \sigma_{1}^{2}}{1+\omega_{1}}+N_{01}\right) \frac{z^{2}}{4}} K_{1}\left(\frac{\sqrt{N_{02}}}{\mathcal{G}_{\mathrm{F}} \sigma_{r d}} z\right) d z\right) .
\end{aligned}
$$

Further, using [13, Eq. (2.16.8.4)] and after some algebraic manipulations, we can express the average BER as

$$
\begin{aligned}
P_{b} & =\frac{1}{2}\left(1-\left(\frac{\left(\bar{\gamma}_{1}+\eta_{1}+1\right) \sqrt{\bar{\gamma}_{1}}}{\sqrt{\pi}\left(\bar{\gamma}_{1}+\frac{\eta_{1}}{1+\omega_{1}}+1\right)^{\frac{3}{2}} \bar{\gamma}_{2}}\right)\right. \\
& \times \sum_{m=0}^{\infty} \frac{\left(-\frac{\omega_{1} \eta_{1}}{1+\omega_{1}}\right)^{m}}{(m !)^{2}} \frac{\Gamma\left(m+\frac{3}{2}\right) \Gamma\left(m+\frac{1}{2}\right)}{\left(\bar{\gamma}_{1}+\frac{\eta_{1}}{1+\omega_{1}}+1\right)^{m}} \\
& \left.\times \Psi\left(m+\frac{3}{2}, 2, \frac{\bar{\gamma}_{1}+\eta_{1}+1}{\left(\bar{\gamma}_{1}+\frac{\eta_{1}}{1+\omega_{1}}+1\right) \bar{\gamma}_{2}}\right)\right) .
\end{aligned}
$$

Note that in the special case of a Rayleigh faded interferer, substituting $\omega_{1}=0$ and $m=0$ into (38) and using

$$
\Psi(a, 2 a-1, x)=\frac{x^{\frac{3}{2}-a} e^{\frac{x}{2}}}{2(a-1) \sqrt{\pi}}\left(K_{a-\frac{1}{2}}\left(\frac{x}{2}\right)-K_{a-\frac{3}{2}}\left(\frac{x}{2}\right)\right)
$$

it can be shown that $P_{b}$ admits the following closed-form solution

$$
P_{b}=\frac{1}{2}\left(1-\frac{1}{2 \bar{\gamma}_{2}} \sqrt{\frac{\bar{\gamma}_{1}}{\bar{\gamma}_{1}+\eta_{1}+1}} e^{\frac{1}{2 \bar{\gamma}_{2}}} \Theta\left(\frac{1}{2 \bar{\gamma}_{2}}\right)\right),
$$

where $\Theta(x)=K_{1}(x)-K_{0}(x)$. 


\section{B. Scenario (b)}

Let us now assume the case of Scenario $(b)$. After demodulation, the decision statistic for $s_{0}$ can be expressed as

$$
\begin{aligned}
\mathcal{D}_{0} & =h_{r d} \mathcal{G}_{\mathrm{F}}\left(\sqrt{P_{s}} h_{s r} s_{0}+\sqrt{P_{1}} h_{1} \cos \theta s_{1}+\tilde{n}_{r}\right) \\
& +\sqrt{P_{2}} h_{2} \cos \left(\theta_{2}-\theta_{r d}-\theta_{s r}\right) s_{2}
\end{aligned}
$$

and the average BER conditioned on $h_{s r}$ can be written as

$$
\begin{aligned}
\widetilde{P}_{b}=\operatorname{Pr}( & \sqrt{P_{s}} h_{s r} s_{0}+\sqrt{P_{1}} h_{1} \cos (\theta) s_{1}+\tilde{n}_{r} \\
& \left.+\frac{\sqrt{P_{2}} h_{2} \cos (\varphi) s_{2}}{h_{r d} \mathcal{G}_{\mathrm{F}}}<0 \mid s_{0}=+1, h_{s r}\right)
\end{aligned}
$$

where $\varphi=\left(\theta_{2}-\theta_{r d}-\theta_{s r}\right)$. Let $\Upsilon=\sqrt{P_{1}} h_{1} \cos (\theta) s_{1}+\tilde{n}_{r}+$ $\frac{\sqrt{P_{2}} h_{2} \cos (\varphi) s_{2}}{h_{r d} \mathcal{G}_{\mathrm{F}}}=I+X_{2}$. By using a similar approach as in Section IV-A we can re-express (42) as

$$
P_{b}=\frac{1}{2}\left(1-\sqrt{\frac{P_{s} \sigma_{s r}^{2}}{\pi}} \int_{0}^{\infty} \Phi_{\Upsilon}(z) e^{-\frac{P_{s} \sigma_{s r}^{2} z^{2}}{4}} d z\right)
$$

where the CF of $\Upsilon$ is expressed as

$$
\begin{aligned}
\Phi_{\Upsilon}(z) & =\Phi_{I}(z) \Phi_{X_{2}}(z) \\
& =e^{-\frac{P_{1} \sigma_{1}^{2} z^{2}}{4\left(1+\omega_{1}\right)}} J_{0}\left(\sqrt{\frac{\omega_{1} P_{1} \sigma_{1}^{2}}{1+\omega_{1}} z}\right) \Phi_{X_{2}}(z) .
\end{aligned}
$$

Moreover, $\Phi_{X_{2}}(z)$ is expressed as

$$
\Phi_{X_{2}}(z)=e^{-\frac{N_{01} z^{2}}{4}} \int_{0}^{\infty} J_{0}\left(\frac{\sqrt{P_{2}} y z}{\mathcal{G}_{\mathrm{F}}}\right) f_{Y}(y) d y
$$

where $Y=\frac{h_{2}}{h_{r d}}$ and $f_{Y}(y)$ is the pdf of the ratio of a Rician/Rayleigh RV. Using the cdf of $h_{2}$ and the pdf of $h_{r d}$ we can express the cdf of $Y$ as

$$
\begin{aligned}
& F_{Y}(y)=\int_{0}^{\infty} F_{h_{2}}(x y) f_{h_{r d}}(x) d x \\
& =1-\frac{2}{\sigma_{r d}^{2}} \int_{0}^{\infty} x Q\left(\sqrt{2 \omega_{2}}, \sqrt{\frac{2\left(1+\omega_{2}\right)}{\sigma_{2}^{2}}} x y\right) e^{-\frac{x^{2}}{\sigma_{r d}^{2}}} d x
\end{aligned}
$$

where $Q(a, b)$ is the Marcum $Q$-function [12, Eq. (1)]. Simplifying (46) using [12, Eq. (40)] and [12, Eq. (41)] yields

$$
F_{Y}(y)=\frac{\left(1+\omega_{2}\right) \sigma_{r d}^{2} y^{2}}{\left(1+\omega_{2}\right) \sigma_{r d}^{2} y^{2}+\sigma_{2}^{2}} e^{-\frac{\omega_{2} \sigma_{2}^{2}}{\left(1+\omega_{2}\right) \sigma_{r d}^{2} y^{2}+\sigma_{2}^{2}}} .
$$

Differentiating (47) with respect to $y$, we obtain the pdf as

$$
\begin{aligned}
f_{Y}(y) & =\frac{2 \omega_{2}\left(1+\omega_{2}\right)^{2} \sigma_{r d}^{4} \sigma_{2}^{2} y^{3}}{\left(\left(1+\omega_{2}\right) \sigma_{r d}^{2} y^{2}+\sigma_{2}^{2}\right)^{3}} e^{-\frac{\omega_{2} \sigma_{2}^{2}}{\left(1+\omega_{2}\right) \sigma_{r d}^{2} y^{2}+\sigma_{2}^{2}}} \\
& +\frac{2\left(1+\omega_{2}\right) \sigma_{r d}^{2} \sigma_{2}^{2} y}{\left(\left(1+\omega_{2}\right) \sigma_{r d}^{2} y^{2}+\sigma_{2}^{2}\right)^{2}} e^{-\frac{\omega_{2} \sigma_{2}^{2}}{\left(1+\omega_{2}\right) \sigma_{r d}^{2} y^{2}+\sigma_{2}^{2}}} .
\end{aligned}
$$

Hence, by substituting (48) into (45), $\Phi_{X_{2}}(z)$ can be evaluated. Unfortunately, however, with $\omega_{2} \neq 0, \Phi_{X_{2}}(z)$ can not be evaluated in closed-form. Nevertheless, using (44) in (43), the average BER can be expressed as

$$
\begin{aligned}
P_{b} & =\frac{1}{2}\left(1-\sqrt{\frac{P_{s} \sigma_{s r}^{2}}{\pi}} \int_{0}^{\infty}\left(\int_{0}^{\infty} J_{0}\left(\frac{\sqrt{P_{2}} y z}{\mathcal{G}_{\mathrm{F}}}\right) f_{Y}(y) d y\right)\right. \\
& \left.\times J_{0}\left(\sqrt{\frac{\omega_{1} P_{1} \sigma_{1}^{2}}{1+\omega_{1}}} z\right) e^{-\left(P_{s} \sigma_{s r}^{2}+\frac{P_{1} \sigma_{1}^{2}}{1+\omega_{1}}+N_{01}\right) \frac{z^{2}}{4}} d z\right) .
\end{aligned}
$$

In the special case where $h_{2}$ is Rayleigh distributed, we can substitute $\omega_{2}=0$ into (47) and next using (45), $\Phi_{X_{2}}(z)$ can be written as

$$
\Phi_{X_{2}}(z)=\frac{2 \sigma_{2}^{2} e^{-\frac{N_{01} z^{2}}{4}}}{\sigma_{r d}^{2}} \int_{0}^{\infty} \frac{y J_{0}\left(\frac{\sqrt{P_{2}} y z}{\mathcal{G}_{\mathrm{F}}}\right)}{\left(y^{2}+\frac{\sigma_{2}^{2}}{\sigma_{r d}^{2}}\right)^{2}} d y .
$$

Using [13, Eq. (2.12.2.28)] and noting that $K_{-1}(x)=K_{1}(x)$, (50) can be evaluated as

$$
\Phi_{X_{2}}(z)=\frac{\sqrt{P_{2}} \sigma_{2}}{\mathcal{G}_{\mathrm{F}} \sigma_{r d}} z e^{-\frac{N_{01} z^{2}}{4}} K_{1}\left(\frac{\sqrt{P_{2} \sigma_{2}^{2}}}{\mathcal{G}_{\mathrm{F}} \sigma_{r d}} z\right) .
$$

Finally, if $h_{1}$ is also Rayleigh distributed, it can be shown that the overall BER takes the form

$$
P_{b}=\frac{1}{2}\left(1-\frac{\eta_{2}}{2 \bar{\gamma}_{2}} \sqrt{\frac{\bar{\gamma}_{1}}{\bar{\gamma}_{1}+\eta_{1}+1}} e^{\frac{\eta_{2}}{2 \bar{\gamma}_{2}}} \Theta\left(\frac{\eta_{2}}{2 \bar{\gamma}_{2}}\right)\right) .
$$

\section{NUMERICAL RESULTS}

In this section, we illustrate the expressions derived in Sections III and IV using numerical examples and examine the effect of interference on the system's performance. It is noted that all results presented here were also verified by simulations.

Fig. 1 depicts the outage probability of dual-hop AF fixed gain relaying for different values of the Rician $K$-factor, $\omega_{1}$, and assuming Scenario (a). The power of the interfering signal at $R$ is assumed to be $20 \mathrm{~dB}$ and $40 \mathrm{~dB}$ lower than the received signal power, while the average SNRs at the $S-R$ and $R$ - $D$ links are assumed equal to each other, so that $\eta_{1}=\bar{\gamma}_{1}-20 \mathrm{~dB}=$ $\bar{\gamma}_{2}-20 \mathrm{~dB}$ and $\eta_{1}=\bar{\gamma}_{1}-40 \mathrm{~dB}=\bar{\gamma}_{2}-40 \mathrm{~dB}$. As can be seen, the outage probability is hardly affected by the Rician $K$-factor of the interferer channel at $R$, yet any increase in $\omega_{1}$ results in a small outage probability increase.

Similar results regarding the outage probability of the fixed gain system under consideration of the Scenario (b) are shown in Fig. 2, where the relative received power of the interferers at both $R$ and $D$ are assumed $20 \mathrm{~dB}$ and $40 \mathrm{~dB}$ lower than the received signal power at $R$ and $D$, respectively. In particular, it is noted that there is hardly any dependence of the outage probability on the Rician $K$-factor of the interferer on the relay, as well as that the achievable diversity order is zero, as was analytically shown in Section III-A2.

Fig. 3 illustrates the BPSK-modulated BER results for both Scenarios $(a)$ and $(b)$. In addition to the aforementioned observations, one may note that in the case of $\eta_{2}=\bar{\gamma}_{1}-20 \mathrm{~dB}$ and for low-to-medium SNRs, there is a cross-over point in the average BER curves of Scenarios $(a)$ and $(b)$. This interesting observation can be explained from the fact that, in the low SNR region, the BER is dominated by the AWGN power. Therefore, 


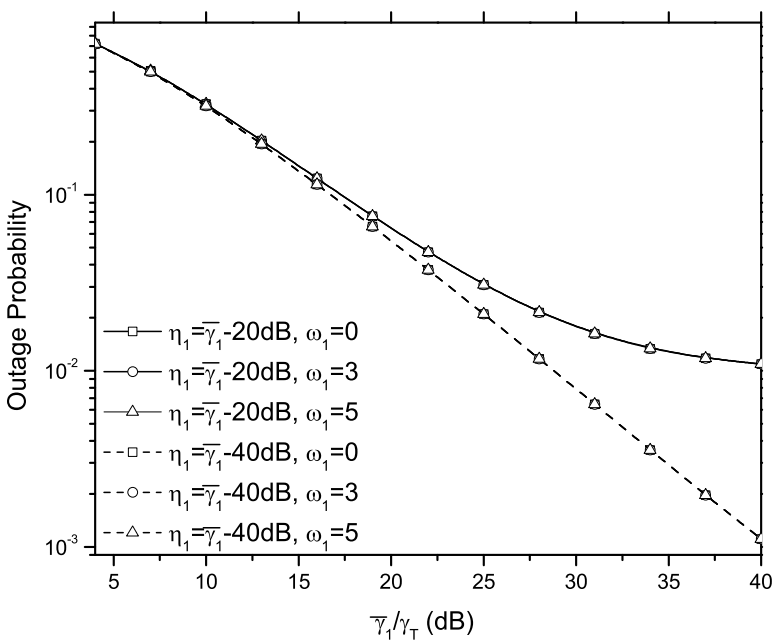

Fig. 1. Outage probability of Scenario (a) for $\eta_{1}=\bar{\gamma}_{1}-20 \mathrm{~dB}=\bar{\gamma}_{2}-20 \mathrm{~dB}$ and $\eta_{1}=\bar{\gamma}_{1}-40 \mathrm{~dB}=\bar{\gamma}_{2}-40 \mathrm{~dB}$.

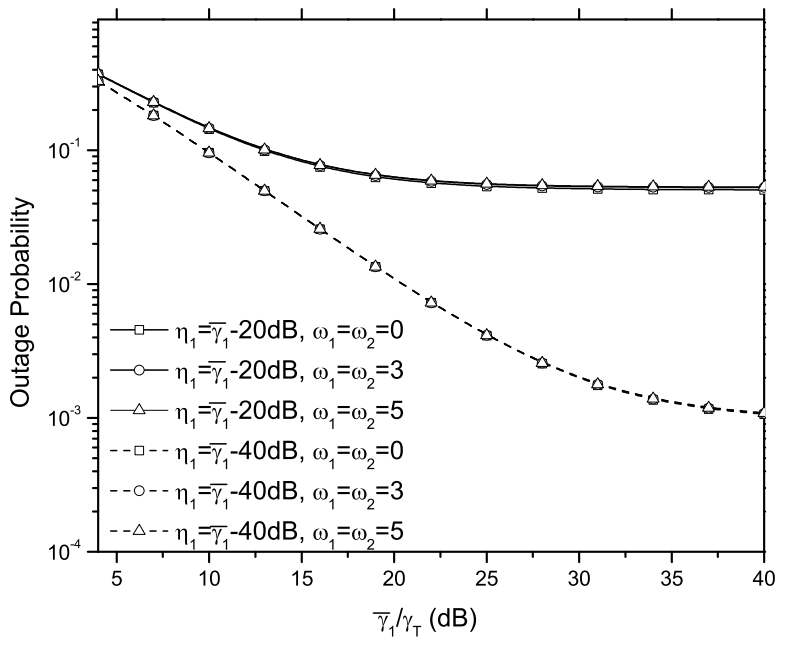

Fig. 2. Outage probability of Scenario $(b)$ for $\bar{\gamma}_{1}=\bar{\gamma}_{2}$ and $\eta_{1}=\eta_{2}=$ $\bar{\gamma}_{1}-20 \mathrm{~dB}$ and $\eta_{1}=\eta_{2}=\bar{\gamma}_{1}-40 \mathrm{~dB}$.

a low interference power, $\left(\eta_{2}=\bar{\gamma}_{1}-20 \mathrm{~dB}\right)$ at $D$ in the case of Scenario $(b)$ can lead to a lower BER, as compared to high AWGN power at $D$ in case of Scenario $(a)$. We also note that the BER cross-over point in the case of $\eta_{2}=\bar{\gamma}_{1}-40 \mathrm{~dB}$ is at $\bar{\gamma}_{1}=40 \mathrm{~dB}$.

\section{CONCLUSIONS}

In this paper, we investigated the performance of a dual-hop fixed gain AF relay system with interference. Both interferencefree and interference-limited reception at the destination were studied by deriving new expressions for the outage probability and, as well as precise average BER expressions. It was shown that, as expected the presence of interference at the relay and/or the destination significantly degrades the performance. Numerical results, which were verified by simulations, revealed that the overall performance is hardly affected by the Rician-K factor of the interfering channel.

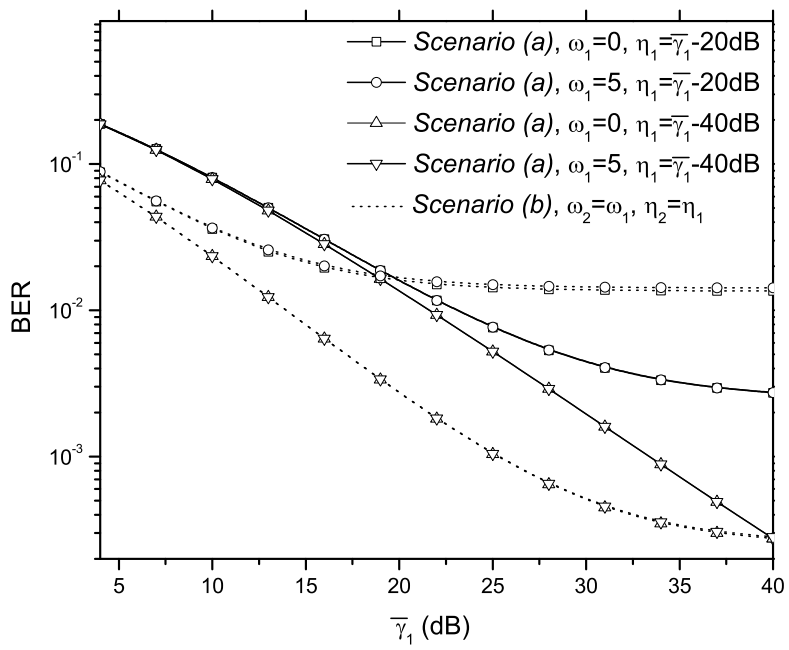

Fig. 3. BER of Scenarios (a) and (b), for $\bar{\gamma}_{1}=\bar{\gamma}_{2}$ and $\eta_{1}=\eta_{2}=\bar{\gamma}_{1}-20 \mathrm{~dB}$ and $\eta_{1}=\eta_{2}=\bar{\gamma}_{1}-40 \mathrm{~dB}$.

\section{REFERENCES}

[1] M. Uysal (Ed.), Cooperative Communications for Improved Wireless Network Transmission: Frameworks for Virtual Antenna Array Applications. IGI-Global, 2009.

[2] M. O. Hasna and M.-S. Alouini, "A performance study of dual-hop transmissions with fixed gain relays," IEEE Trans. Wireless Commun., vol. 3, pp. 1963-1968, Nov. 2004.

[3] D. Senaratne and C. Tellambura, "Unified exact performance analysis of two-hop amplify-and-forward relaying in Nakagami fading," IEEE Trans. Veh. Technol., vol. 59, pp. 1529-1534, Mar. 2010.

[4] I. Krikidis, J. S. Thompson, S. Mclaughlin and N. Goertz, "Max-min relay selection for legacy amplify-and-forward systems with interference," IEEE Trans. Wireless Commun., vol. 8, pp. 3016-3027, June 2009.

[5] A. Agustin and J. Vidal, "Amplify-and-forward cooperation under interference-limited spatial reuse of the relay slot," IEEE Trans. Wireless Commun., vol. 7, pp. 1952-1962, May 2008.

[6] Q. Yang, K. S. Kwak and F. Fu, "Closed-form expression for outage probability of DF relaying with unequal Nakagami interferers in Nakagami fading," in Proc. IEEE PIMRC 2009, Tokyo, Japan, pp. 335-339.

[7] D. Lee, and J. H. Lee "Outage probability for opportunistic relaying on multicell environment," in Proc. IEEE VTC Spring 2009, pp. 1-5.

[8] C. Zhong, S. Jin and K-K. Wong, "Dual-hop systems with noisy relay and interference-limited destination," IEEE Trans. Commun., vol. 58, pp. 764-768, Mar. 2010.

[9] H. A. Suraweera, H. K. Garg and A. Nallanathan, "Performance analysis of two hop amplify-and-forward systems with interference at the relay," IEEE Commun. Lett., vol. 14, pp. 692-694, Aug. 2010.

[10] S. Chen, X. Zhang, F. Liu and D. Yang, "Outage performance of dual-hop relay network with co-channel interference," in Proc. IEEE VTC Spring 2010, Taipei, Taiwan, May 2010, pp. 1-5.

[11] I. S. Gradshteyn and I. M. Ryzhik, Table of Integrals, Series and Products, 7th ed., New York: Academic, 2007.

[12] A. H. Nuttall, "Some integrals involving the $Q$-function," Naval Underwater Systems Center (NUSC) Technical Report 4297, April 1972.

[13] A. P. Prudnikov, Y. A. Brychkov and O. I. Marichev, Integrals and Series. vol. 2, New York: NY, Gordon and Breach Science Publishes, 1986.

[14] M. Abramowitz and I. A. Stegun, Handbook of Mathematical Functions with Formulas, Graphs, and Mathematical Tables, 9th ed. New York: Dover, 1970.

[15] N. C. Beaulieu and J. Cheng, "Precise error-rate analysis of bandwidthefficient BPSK in Nakagami- $m$ fading and cochannel interference," IEEE Trans. Commun., vol. 52, pp. 149-158, Jan. 2004.

[16] M. B. Parsley, "Performance evaluation for phase-coded spread-spectrum multiple-access communication - Part I," IEEE Trans. Commun., vol. COM-25, pp. 795-799, Aug. 1977.

[17] A. Papoulis, The Fourier Integral and its Applications, New York: McGraw-Hill, 1962. 